\title{
Evitapp: Persuasive Application for Physical Activity and Smoking Cessation ${ }^{\dagger}$
}

\author{
Antonio Bascur ${ }^{1}$ (1) , Pedro O. Rossel ${ }^{1, *(1)}$, Valeria Herskovic ${ }^{2}$ (i) and \\ Claudia Martínez-Carrasco ${ }^{3,4}$ (D) \\ 1 Department of Computer Science, Universidad Católica de la Santísima Concepción, Concepción 4090541, \\ Chile; abascur@ing.ucsc.cl \\ 2 Department of Computer Science, Pontificia Universidad Católica de Chile, Santiago 7820436, Chile; \\ vherskov@ing.puc.cl \\ 3 Faculty of Medicine, Universidad Católica de la Santísima Concepción, Concepción 4090541, Chile; \\ camartinez@ucsc.cl or claudiamartinez04@gmail.com \\ 4 Faculty of Health Sciences, Universidad San Sebastián, Concepción 4080871, Chile \\ * Correspondence: prossel@ucsc.cl; Tel.: +56-41-234-5322 \\ + Presented at the 12th International Conference on Ubiquitous Computing and Ambient Intelligence \\ (UCAmI 2018), Punta Cana, Dominican Republic, 4-7 December 2018.
}

Published: 30 October 2018

\begin{abstract}
The most important risk factors for cardiovascular health are smoking and a sedentary lifestyle. This paper proposes Evitapp, a mobile application designed to promote physical activity and smoking cessation. The application does not use additional tracking devices, rather relying on phone sensors to track physical activity, and on users logging their behavior. Nineteen users tested the application over 10 days. Participants found the applications easy to use and used them approximately once per day. Even though the habits of the experiment participants did not change significantly, those who used the smoking cessation application reported decreasing their smoking habit.
\end{abstract}

Keywords: persuasive technology, healthy lifestyle, sedentarism, smoking cessation

\section{Introduction}

Cardiovascular disease is a public health problem, and it is the leading cause of death and disability worldwide [1]. Cardiovascular disease is considered an umbrella term, under which it is possible to find a group of pathologies, e.g., coronary heart disease, cerebrovascular disease, and peripheral arterial disease [2].

The concept of cardiovascular risk factors is applied to those biological signs and acquired habits that increase the probability of having a cardiovascular disease [3]. These can be found in all types of population, at all socioeconomic levels [2] The concept of primary prevention appears is relevant in people with risk factors who do not have symptoms of cardiovascular disease [4]. This prevention is described as effective since it is capable of eliminating risky behaviors, making it possible to prevent at least $80 \%$ of cardiovascular diseases [5].

Within the control strategies, self-monitoring appears as a way to change related lifestyles, promoting positive behaviors, e.g., smoking cessation, weight loss, physical activity [1]. The most important risk factors for their adverse effect on the cardiovascular system are smoking and sedentary lifestyle [6].

Regarding smoking, most smokers feel dissatisfied with their smoking habit and although they would like to quit, they tend to overestimate how likely they are to actually do so [7]. Even though 
there is no level of safe consumption, when consumption is lower, this lowers the risk of suffering a cardiovascular disease [8].

Regarding physical activity, sedentary lifestyle is a behavior in which the subject does not perform physical activity at least three times a week for at least 30 min during the last month [9]. Sedentary behavior doubles the probability of dying from coronary heart disease [10].

Persuasive technologies are those designed to change a person's behavior in some way [11]. To perform a desired behavior, a person must be able, motivated, and triggered to do so [12]. Persuasive technologies may have a large impact on the health and well-being of populations. Some application domains for persuasive technologies are improving behaviors related to smoking, TV and internet use, stress management, social relationships, sexual behavior, exercise, diet, education, and environmental conservation [13]. There are several strategies for persuasive technologies, e.g., instruction style (authoritative or non-authoritative), social feedback (cooperative or competitive), motivation (extrinsic or intrinsic) and reinforcement type (negative or positive) [14].

This paper proposes a mobile application, called Evitapp, designed to help control cardiovascular risk factors. This application does not use additional tracking devices, rather relying on the mobile phone's available sensors: accelerometer and GPS, to track physical activity, and on the user's logging of their behavior. The application has two different versions: one to promote smoking cessation, and one to promote physical activity, with a high software reuse between both. This paper presents a study in which two different groups of users tested Evitapp, with one group testing the smoking cessation version and the other testing the physical activity version.

This paper is organized as follows. First, we discuss related work, considering persuasive technologies for physical activity and smoking cessation. Then, we describe the design and implementation of our applications. Section 4 describes our methodology, then Section 5 describes the results and Section 6 presents the discussion. Finally, Section 7 presents our conclusions and discusses possible paths of future work.

\section{Related Work}

\subsection{Persuasive Technologies for Physical Activity}

A systematic review of persuasive applications to promote physical activity found that persuasive features could be categorized as pertaining to the primary task, dialogue support, system credibility support, and social support [15]. Within the primary task, self-monitoring-either manual or automatic (e.g., through activity trackers)—was the most used design feature [15]. A review of applications available in 2015 on iTunes and Google Play found that applications most often included user input, textual or numerical views of behavior and progress, sharing on social networks, and general advice, including less often such features as gamification and expert contact [16].

Many mobile applications to promote physical activity have been proposed in the literature. Several use additional devices, such as activity or personal health trackers. For example, a mobile application called Heart Angel used a mobile phone, paired with a heart rate monitor and a GPS, allowed users to assess and monitor their fitness level [17]. Another example of this trend is a mobile application called Active Coach, which is paired with a Fitbit tracker [18]. Active Coach was developed specifically for working young adults with low education, by choosing determinants to promote active lifestyle and using evidence-based behavior change techniques to convert them into practical applications [18]. The incorporation of target users into the development process was found to be an important aspect of developing an application that considered user preferences [18]. A mobile application called SitCoach monitored physical activity and prompted users to take active breaks through messages, finding that simple reminders sufficed to trigger users to take breaks [19].

UbiFit Garden [20] uses a garden (with only positive reinforcement) as a representation on the mobile phone wallpaper of the goals users reach; providing a reminder every time the phone is used. 


\subsection{Persuasive Technologies for Smoking Cessation}

A wealth of research exists regarding smoking cessation programs and initiatives. Overall, picture warnings, ad bans, and increased taxes are public policies that have been shown to reduce tobacco use [21]. The persuasive effects of anti smoking messages have been studied, finding, e.g., that messages stressing gains (over losses) and short-term (over long-term) consequences have more effects on smoker attitudes-e.g., a message stressing that quitting smoking improves stamina [22]. Many mobile applications use reminders in the form of messages-e.g., SMS and MMS [23]. Some studies have focused on how mobile applications incorporating persuasive messages may decrease boredom and annoyance with the messages users receive-e.g., by using diverse triggers, such as those that use concepts related to the self [24].

Many mobile applications to support smoking cessation efforts exist, however, those with scientific research backing them may be difficult to find [25]. On the other hand, technology may be evolving at a faster pace than that of scientific publication [25]. A review of smoking cessation applications available in the iTunes store and Google Play, according to the self-determination theory, found that few applications addressed autonomy, competence and relatedness, and that in general, applications do not sufficiently stimulate autonomous motivation [26].

A user-centered design process is helpful to design a smoking tracking application that allows users to easily track behavior [27]. A qualitative study on what information users would like from a text message service for smoking cessation found that users would like to feel involved with the program, communicate their feelings and visualize their progress [28].

\section{Evitapp: Design and Implementation}

\subsection{Persuasive Strategies and Mockups}

In this work, we developed two applications: one for physical activity and other one for smoking cessation. These were implemented in Spanish, because the applications were to be tested in Concepción, Chile.

To develop the applications, we started by defining the persuasive strategies. Based on a review of persuasive technologies for health and wellness [29], as well as specific research concerning physical activity [30-32] and smoking cessation [33-35], and the review of existing applications from Google Play [36,37], we decided to incorporate the following five persuasive strategies (1) tracking and monitoring, (2) social support, sharing, and comparison, (3) persuasive messages, reminders, and alerts, (4) goals and objective, and (5) emoticons and persuasive images.

With the previous in mind, we first used NinjaMock (https://ninjamock.com) to build some mockups of the software for smoking cessation. We used the metaphor of a tree in a field (Figure 1a) to visualize goals and objectives, and provide persuasive images. This image would change proportionally with the user's progress, adding landscape elements (Figure 1b). Initially, it has few elements, and as the user avoids cigarettes or increases their physical activity, both the percentage and the number of elements in the landscape increases, making it more cheerful and abundant in terms of number of animals, clouds, etc. The field was also the backdrop for the complete application, allowing the end-user to interact with a harmonious environment. 


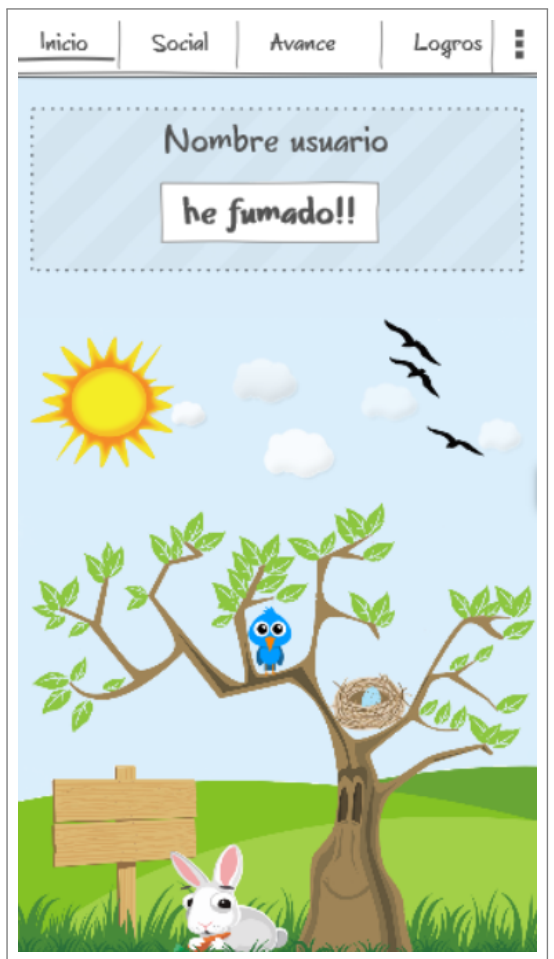

(a) Main Window

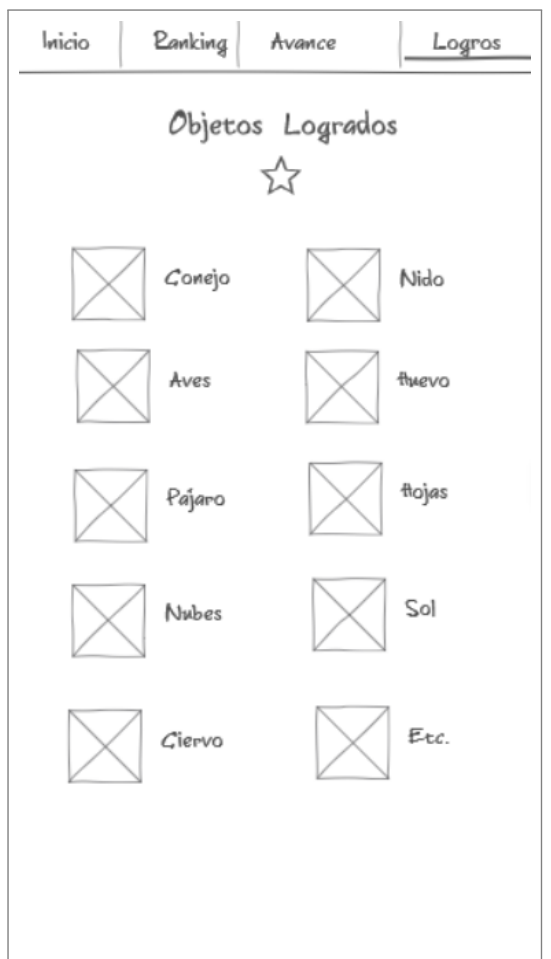

(b) Landscape Elements

Figure 1. First version Mockups of Evitapp

\subsection{Final Applications}

We started with the development of the smoking cessation application. This was developed using Android Studio 3.0, and for a version of Android 4.4 (KitKat) and higher.

Figure 2a displays the sign up form for the application. Users must input the number of cigarettes they smoke daily (¿Cuántos cigarros fuma cada día?). This question is important because this data is used to calculate the user's progress. Figure $2 b$ displays the main window of the application, where the central element is the tree in a field metaphor representing the user's progress, which is in this case $97.58 \%$. In the upper part of the application, it displays the name and image of the user, the number of cigarettes smoked in the current day, along with the rate of progress. Two buttons are used to report cigarette smoking, either increasing or decreasing the number of cigarettes that have been smoked in that day.

The social aspects of the persuasive strategies were implemented as a social network, where all people that have the same application can see the tree in a field metaphor of the others. Figure 3a shows the social tab, which contains a list of all users that use the application, along with their picture and occupation. If a user is selected from the list, the current image of his/her tree will be displayed, allowing to see his/her progress.

Finally, Figure $3 \mathrm{~b}$ shows all possible elements to be displayed in the landscape, in order of achievement. Each element represents a goal to be reached by the user. When selecting any of the elements, a detail of the achievement, equivalent advance percentage and the corresponding number of cigarettes will be shown. 


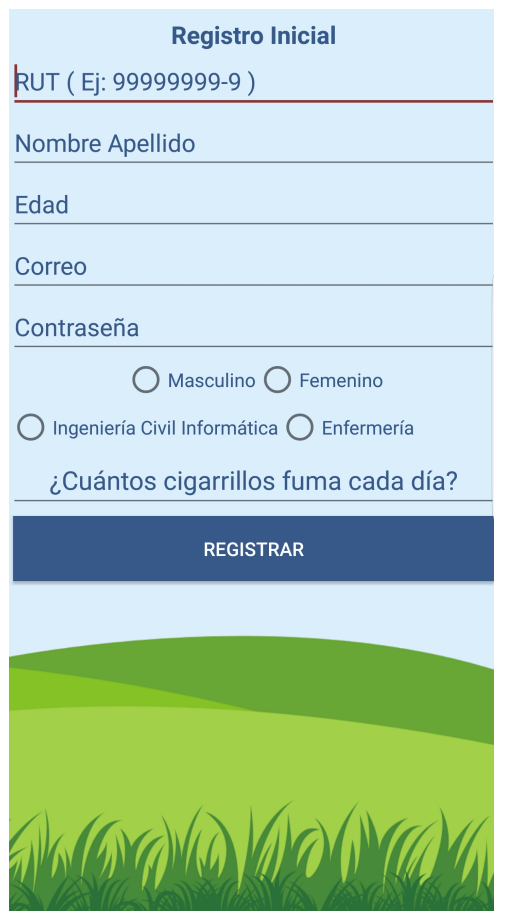

(a) User Register

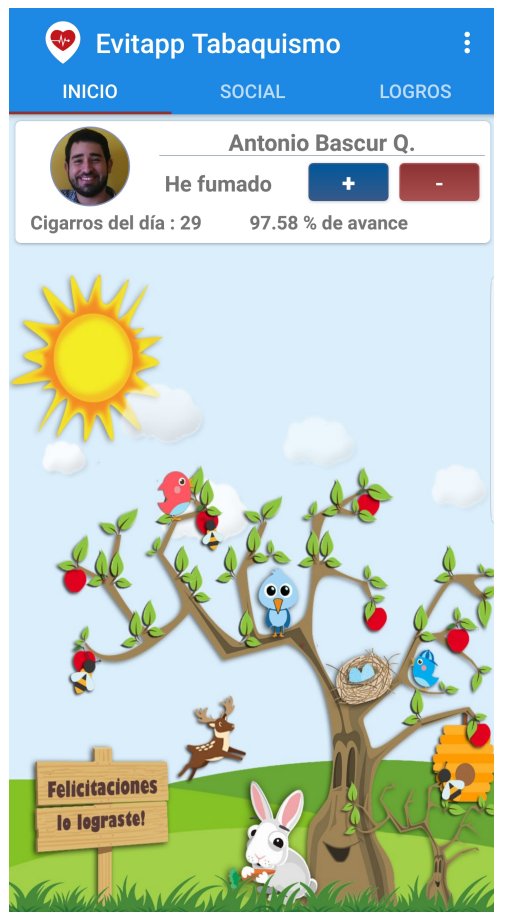

(b) Main Window

Figure 2. Evitapp Final Version: Smoking Cessation.

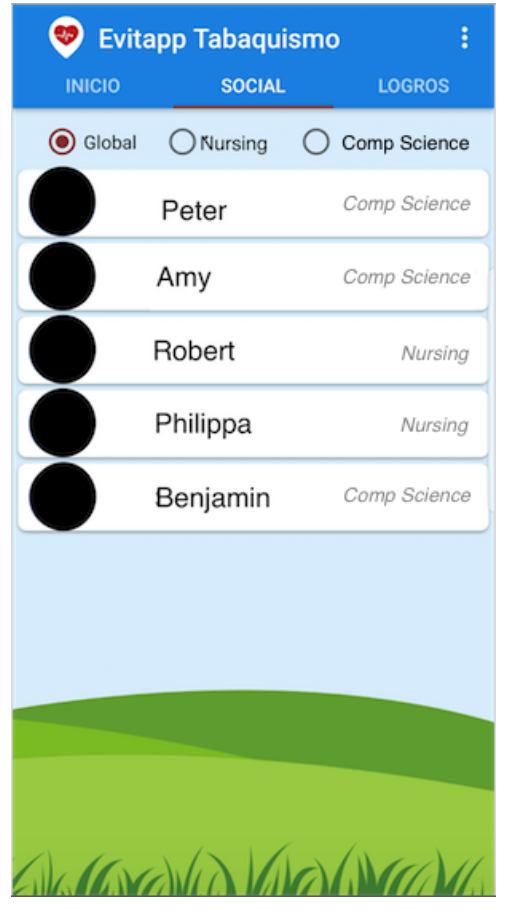

(a) Social

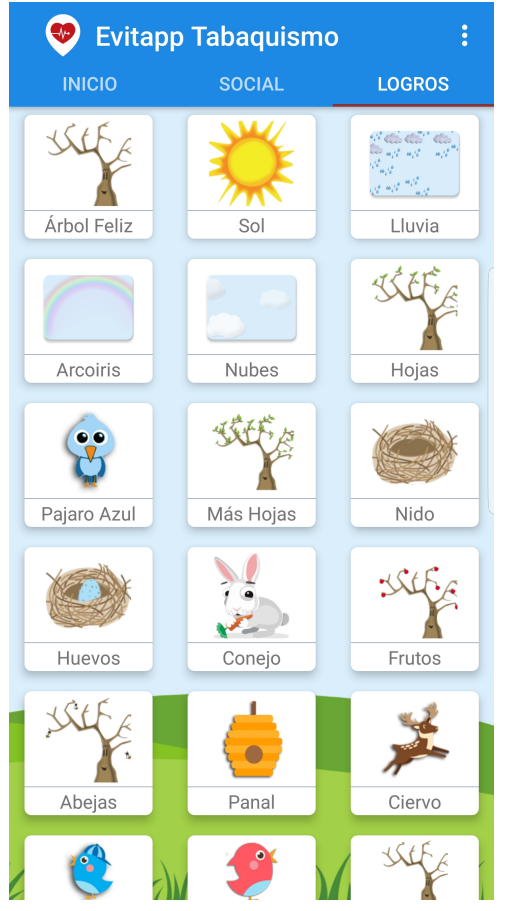

(b) Achievements

Figure 3. Evitapp Final Version: Smoking Cessation (continuation).

The sign up form for the physical activity application in which the initial question is how many minutes of physical activity the user does in a week (¿Minutos de actividad física a la semana?). In the main window, the application has two buttons, one blue and the other red, for reporting the activities done during the day, as well as for how long they performed them. When the user reports the activities, 
he/she has to choose from a list of options (Figure 4a). Users can choose to see other users, along with their progress in metaphor form (Figure $4 b$ ).

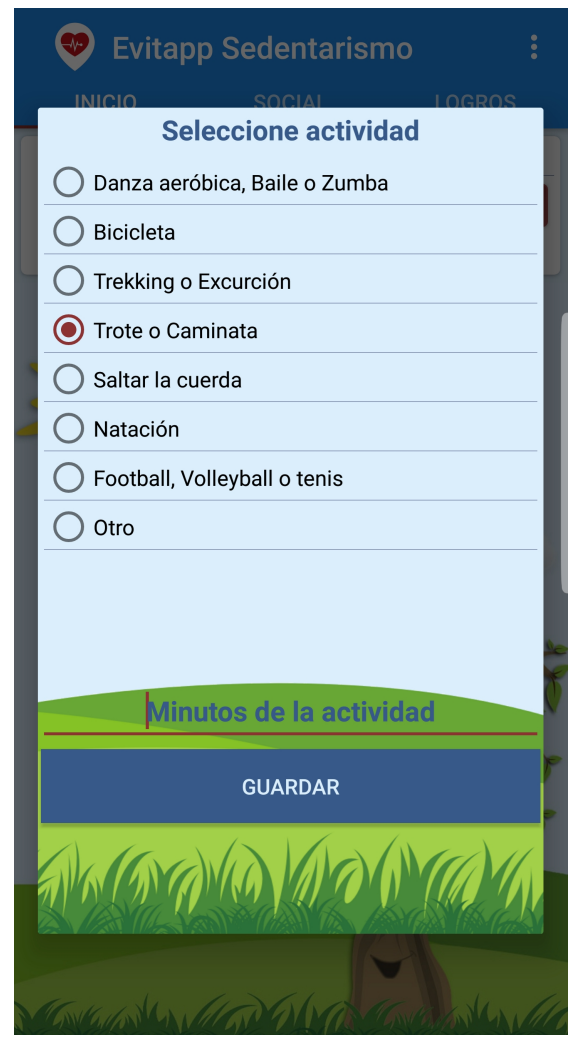

(a) Physical Activity Register

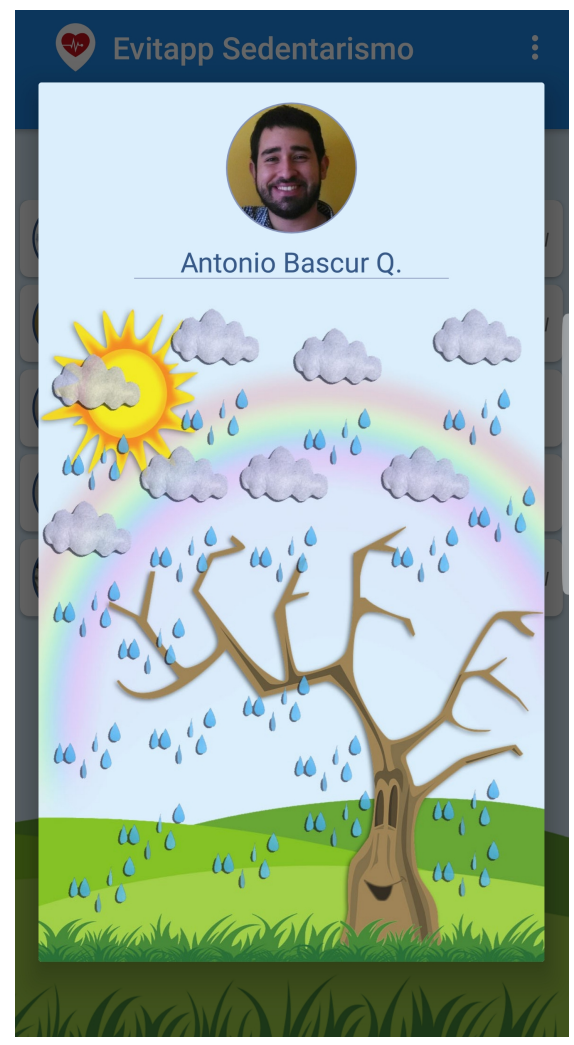

(b) User Landscape

Figure 4. Evitapp Final Version: Physical Activity

The application can automatically detect physical activity (specifically walking, jogging or cycling) by using the GPS and accelerometer available in the mobile device. This feature was implemented using the Activity Recognition API developed for Android by Google [38]. The application counts the number of continuous minutes of activity. When the activity is greater than $10 \mathrm{~min}$ those minutes are added to the daily register.

Finally, we implemented several notifications in both applications, using a non-diverse strategy [24] to avoid overwhelming users. Figure 5a presents a notification where the application indicates that it has detected twenty-six minutes of walking, jogging or cycling, while Figure $5 \mathrm{~b}$ shows a message that indicates benefits of performing physical activity (Exercise is good for the mind, body and soul). The motivational notifications are shown at random times, with a daily limit of possible notifications.

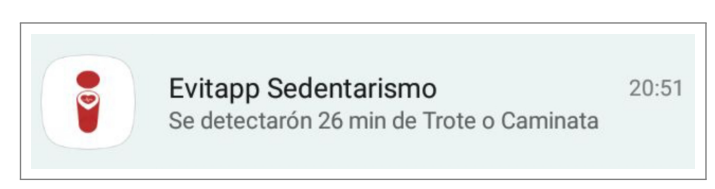

(a) Automatic Detection of Walking, Jogging or Cycling

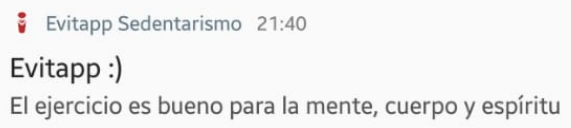

(b) Persuasive Notification Recommending Physical Activity

Figure 5. Examples of Notifications from the Smoke Cessation Application 


\section{Methodology}

\subsection{Data Collection}

We collected participant responses from three standard questionnaires, selected to measure nicotine dependence (Fagerström Test), digital skills (DIGCOMP), and the usability of the application (SUS). Each instrument is described below:

- Fagerström Test is an instrument for measuring physical nicotine dependence. The test has six questions, and the results indicate whether users have low, moderate, or high dependence [39].

- DIGCOMP is an instrument to measure digital competences, i.e., set of knowledge, skills and attitudes needed to be functional in a digital environment. Users are categorized into one of four possible groups, according to their digital skill levels: none, low, basic or above basic [40].

- System usability scale (SUS) is a Likert Scale which includes 10 questions and it permits quickly to measure the usability of the system. In this, scores over 80 indicate very good usability, while scores below 60 indicate poor usability [41].

Furthermore, we built an ad-hoc questionnaire for getting information from the users about the use and perception of the applications (see Table 1).

Table 1. Evitapp questionnaire.

\begin{tabular}{ll}
\hline & Item \\
\hline 1 & It is easy to input an activity/cigarette \\
2 & It is easy to remove an activity/cigarette \\
3 & The tree image represented my progress accurately \\
4 & The application was available when I wanted to use it \\
5 & I was always notified of any change in my tree image \\
6 & The images used for each element were adequate \\
7 & The application notified me daily to remind me to use it \\
8 & I liked being able to see the trees for other members of the group \\
9 & I liked being able to see the details for each element in the tree image \\
10 & The way to input information in a day that I missed is simple \\
\hline
\end{tabular}

\subsection{Participants}

Our participants were nineteen adult people ( 7 women, 12 men), with an average age of 29 years (SD:10.4). Ten of them were university students, and the rest had other occupations. Each participant signed an inform consent form for participating in the experiment.

Table 2 summarizes our study participants, displaying the age and gender of each, as well as which application (Evitapp for Smoking cessation or Evitapp for Physical Activity, or both) they used during the experiment (marked as $\bullet$ ), their level of digital skills according to the DIGCOMP questionnaire, and the level of physical activity (IPAQ) for the participants who used Evitapp for Physical Activity. All users had low dependency to smoking, per the Fagerström Test.

\subsection{Experiment}

To evaluate the applications, each participant used the application, either for physical activity or smoking cessation, for 10 days. In the case of the participants that evaluated both apps, they used both in the same 10 days. The evaluation was conducted during January 2018.

First, one researcher gave a brief introduction about the study and its purpose and the participants completed the DIGCOMP test, Fagerström and IPAQ questionnaires. Participants signed the informed consent form and the researcher installed the application on the participants' own mobile phone.

Participants used the application freely for 10 days, with no intervention from the researchers. They received daily notifications at random times to remind them to use the application. After 
the 10 days, the researcher met with the participants again, and the participants filled out the SUS, Fagerström and IPAQ questionnaires, as well as the Evitapp questionnaire (see Table 1) and answered two questions: whether they felt Evitapp helped them change their habits, and whether they wanted to comment on any positive or negative aspects of the application.

Table 2. Description of study participants.

\begin{tabular}{ccccccc}
\hline P & Age & G & \multicolumn{2}{c}{ Used Application } & Digital Skills & IPAQ \\
\hline Phys. & Smok. & & \\
\hline P1 & 25 & M & $\bullet$ & & Above Basic & Moderate \\
P2 & 25 & M & $\bullet$ & & Above Basic & Low \\
P3 & 24 & M & $\bullet$ & & Above Basic & Moderate \\
P4 & 24 & F & $\bullet$ & & Above Basic & Moderate \\
P5 & 25 & F & $\bullet$ & & Above Basic & Moderate \\
P6 & 22 & F & $\bullet$ & & Basic & Moderate \\
P7 & 53 & M & $\bullet$ & & Basic & Low \\
P8 & 24 & M & $\bullet$ & $\bullet$ & Above Basic & Low \\
P9 & 25 & F & & $\bullet$ & Above Basic & \\
P10 & 25 & M & $\bullet$ & $\bullet$ & Above Basic & Moderate \\
P11 & 26 & F & & $\bullet$ & Above Basic & \\
P12 & 26 & M & & $\bullet$ & Above Basic & \\
P13 & 28 & M & & $\bullet$ & Above Basic & \\
P14 & 31 & M & & $\bullet$ & Above Basic & \\
P15 & 23 & M & & $\bullet$ & Above Basic & \\
P16 & 57 & F & $\bullet$ & $\bullet$ & Above Basic & Low \\
P17 & 20 & M & $\bullet$ & $\bullet$ & Above Basic & Low \\
P18 & 45 & F & $\bullet$ & $\bullet$ & Above Basic & Moderate \\
P19 & 27 & M & & $\bullet$ & Above Basic & \\
\hline
\end{tabular}

\section{Results}

\subsection{User Experience}

On average, participants used the application 13.6 times over the course of the 10-day experiment, where one user only used it once, and three users used it over 25 times.

The SUS scores for each application are presented in Table 3. All user groups rated Evitapp usability to be above average, and users of the physical activity application were the most positive users. We also averaged the answers on the Evitapp questionnaire (see Table 1), finding that overall, users were happy with the application, scoring it over 4.3 (out of 5). To evaluate the internal consistency (reliability) of the Evitapp questionnaire, we used Cronbach's alpha [42]. The value for our questionnaire was 0.81 (good) [43].

Table 3. SUS and Evitapp questionnaire scores for participant groups.

\begin{tabular}{lll}
\hline & SUS & Evitapp \\
\hline Smoking Cessation & 86.4 & 4.3 \\
Physical Activity & 90 & 4.6 \\
Both-Smoking & 83.5 & 4.4 \\
Both-Physical & 87 & 4.4 \\
\hline
\end{tabular}

\subsection{Evitapp Persuasion}

Overall, out of the 12 users that tested the physical activity application (7 tested only this application and 5 tested both), 5 users reported increasing their activity levels, 3 users reported decreasing activity levels, and 4 reported no change. 
Regarding the smoking application, for users that used both applications, there was no reported change in smoking habits (all 5 users reported exactly the same habits in the pre- and post-experiment questionnaires). However, for the 7 users that only used the smoking cessation application, average Fagerström questionnaire results decreased from 0.6 to 0.1 .

\subsection{Qualitative Results}

Participants were asked whether they felt Evitapp helped them change their habits. This section discusses these qualitative results, providing quotes from participants (translated from Spanish).

The responses from participants who tested the smoking cessation application may be categorized into three distinct reasons. First, some users felt the application helped them remember not to smoke. One said, "Yes, it is very interactive and helped me remember that I don't have to smoke constantly without realizing it". Second, one user thought it helped him/her calculate how much he/she was spending on smoking: "It helped me calculate how many cigarettes I smoke per month and realize how much I'm spending on it". Finally, some users commented on the persuasive aspects of the application: "The images help me want to achieve the goals", "The messages that I received helped". Some participants felt the application did not help them change their habits, only realize or confirm how much they smoked.

The responses from participants who tested the physical activity application follow more or less the same pattern as the ones from the smoking application. Some participants liked being reminded to move: "It reminded me constantly that I had to get moving". Some participants thought the application did not change their habit because they are not able to, for lack of time or being busy with other activities: "I was too busy working and could not do more physical activity". Several participants mentioned that the experiment allowed them to realize they were not as sedentary as they had previously thought. One participant also mentioned that the application was good at reminding her to move, without being annoying.

\section{Discussion}

We created a mobile application to persuade users to change their habits, focused on improving cardiovascular health. Participants used the application for 10 days, reporting on their smoking and activity habits. Without being asked about persuasive aspects directly, several users pointed to the persuasive elements (tracking, goals, images, notifications) as helping them use the application and change their habits. The social network feature was not mentioned as important, possibly because the users were strangers among themselves.

Evitapp does not use additional activity trackers (becoming, therefore, a lower-cost alternative than other similar applications, e.g., [17]), relying on the mobile phone's sensors to detect activity. The application uses positive reinforcement, as UbiFit Garden [20], to promote positive behaviors and not discourage users who do not change their behaviors.

There are several limitations that we would like to acknowledge. First, several participants used both applications, which may affect their perception and their usage. For this reason, the analysis of the results considered separately those users who used one application or both. Second, the change in habits was self-reported by participants, who filled out pre- and post-experiment questionnaires. Participants may have been trying to please the researchers or feel that they had accomplished a change, and not been entirely accurate in their reports. An objective way to measure changes would have to be implemented in future experiments. Furthermore, changes in habits, especially in smoking, require a longer period of intervention. Additionally, all participants had a low dependency to smoking, so effects would only be moderate at best. Finally, the experiment was of a short duration and with a limited number of participants, so it is not possible to generalize our findings to a general population or a longer period of time. For this reason as well, although we analyzed whether demographic data (e.g., gender) or digital skills were related to how persuasive the applications were, the number of participants does not make it possible to find clear correlations or trends. 


\section{Conclusions}

This paper presented Evitapp, a set of two applications, one created to persuade users to stop smoking, and the other to promote physical activity. The applications use a tree in a field metaphor, in which as users progress in their journey, the tree is populated with leaves, animals, and other positive elements. The applications were evaluated by 19 users during 10 days. Both applications were positively evaluated by the users, who found them easy to use and used them approximately once per day. However, the applications did not significantly change the habits of the experiment participants. The persuasive effect was most noted in those participants who used the smoking cessation application. These participants reported decreasing their smoking habit. A long term evaluation should be conducted to review whether these results are maintained over time. This paper contributes to literature aimed at building persuasive mobile applications to improve health and prevent disease, by presenting a new application and insights from its evaluation, that may help other researchers create more effective persuasive applications.

Funding: This research was funded by grant DINREG 05/2017 from the Dirección de Investigación e Innovación of the Universidad Católica de la Santísima Concepción, and by CONICYT/FONDECYT 1181162 (Chile).

\section{References}

1. Piette, J.D.; List, J.; Rana, G.K.; Townsend, W.; Striplin, D.; Heisler, M. Mobile Health Devices as Tools for Worldwide Cardiovascular Risk Reduction and Disease Management. Circulation 2015, 132, $2012-2027$. doi:10.1161/CIRCULATIONAHA.114.008723.

2. Stewart, J.; Manmathan, G.; Wilkinson, P. Primary prevention of cardiovascular disease: A review of contemporary guidance and literature. JRSM Cardiovasc. Dis. 2017, 6, 1-9. doi:10.1177/2048004016687211.

3. Cruz-Sánchez, E.; Orosio-Méndez, M.; Cruz-Ramírez, T.; Bernardino-García, A.; Vásquez-Domínguez, L.; Galindo-Palma, N.; Grajales-Alonso, I. Cardiovascular risk factors among nursing students of a public university. Enferm. Univ. 2016, 13, 226-232. doi:10.1016/j.reu.2016.09.004.

4. World Health Organization. Prevención de las enfermedades cardiovasculares. Guía de bolsillo para la estimación y el manejo del riesgo cardiovascular. Available online: http:/ /www.who.int/publications/list/ PocketGL_spanish.pdf (accessed on 8 May 2018). (In Spanish)

5. Piepoli, M.F.; Hoes, A.W.; Agewall, S.; Albus, C.; Brotons, C.; Catapano, A.L.; Cooney, M.T.; Corrà, U.; Cosyns, B.; Deaton, C.; et al. 2016 European Guidelines on cardiovascular disease prevention in clinical practice. Atherosclerosis 2016, 252, 207-274. doi:10.1016/j.atherosclerosis.2016.05.037.

6. Yusuf, S.; Hawken, S.; Ônpuu, S.; Dans, T.; Avezum, A.; Lanas, F.; McQueen, M.; Budaj, A.; Pais, P.; Varigos, J.; et al. Effect of potentially modifiable risk factors associated with myocardial infarction in 52 countries (the INTERHEART study): Case-control study. Lancet 2004, 364, 937-952. doi:10.1016/S0140-6736(04)17018-9.

7. Jarvis, M.J.; McIntyre, D.; Bates, C. Effectiveness of smoking cessation initiatives: Efforts must take into account smokers' disillusionment with smoking and their delusions about stopping. BMJ 2002, 324, 608.

8. Ryan, H.; Trosclair, A.; Gfroerer, J. Adult Current Smoking: Differences in Definitions and Prevalence Estimates-NHIS and NSDUH, 2008. J. Environ. Public Health 2012, 2012, 1-11. doi:10.1155/2012/918368.

9. Martínez, M.A.; Leiva, A.M.; Sotomayor, C.; Victoriano, T.; Von Chrismar, A.M.; Pineda, S. Cardiovascular risk factors among university students. Rev. Méd. Chile 2012, 140, $426-435$. doi:10.4067/S0034-98872012000400002.

10. Fernández-Abascal, E.; Martín-Díaz, M.D.; Domínguez-Sánchez, F.J. Factores de riesgo e intervenciones psicológicas eficaces en los trastornos cardiovasculares. Psicothema 2003, 15, 615-630.

11. Fogg, B.J. Persuasive technologies. Commun. ACM 1999, 42, 27-29.

12. Fogg, B. A Behavior Model for Persuasive Design. In Proceedings of the 4th International Conference on Persuasive Technology; ACM: New York, NY, USA, 2009; pp. 40:1-40:7. doi:10.1145/1541948.1541999.

13. IJsselsteijn, W.; de Kort, Y.; Midden, C.; Eggen, B.; van den Hoven, E. Persuasive Technology for Human Well-being: Setting the Scene. In Proceedings of the First International Conference on Persuasive Technology for Human Well-Being; Springer-Verlag: Berlin/Heidelberg, Germany, 2006; pp. 1-5. 
14. Halko, S.; Kientz, J.A. Personality and Persuasive Technology: An Exploratory Study on Health-Promoting Mobile Applications. In Persuasive Technology; Ploug, T., Hasle, P., Oinas-Kukkonen, H., Eds.; Springer-Verlag: Berlin/Heidelberg, Germany, 2010; pp. 150-161.

15. Matthews, J.; Win, K.T.; Oinas-Kukkonen, H.; Freeman, M. Persuasive Technology in Mobile Applications Promoting Physical Activity: A Systematic Review. J. Med. Syst. 2016, 40, 72:1-72:13.

16. Mollee, J.S.; Middelweerd, A.; Kurvers, R.L.; Klein, M.C.A. What technological features are used in smartphone apps that promote physical activity? A review and content analysis. Pers. Ubiquitous Comput. 2017, 21, 633-643. doi:10.1007/s00779-017-1023-3.

17. Garcia Wylie, C.; Coulton, P. Persuasive Mobile Health Applications. In Electronic Healthcare; Weerasinghe, D., Ed.; Springer-Verlag: Berlin/Heidelberg, Germany, 2009; pp. 90-97.

18. Simons, D.; Bourdeaudhuij, I.D.; Clarys, P.; Cocker, K.D.; Vandelanotte, C.; Deforche, B. A Smartphone App to Promote an Active Lifestyle in Lower-Educated Working Young Adults: Development, Usability, Acceptability, and Feasibility Study. JMIR mHealth uHealth 2018, 6, e44. doi:10.2196/mhealth.8287.

19. van Dantzig, S.; Geleijnse, G.; van Halteren, A.T. Toward a persuasive mobile application to reduce sedentary behavior. Pers. Ubiquitous Comput. 2013, 17, 1237-1246. doi:10.1007/s00779-012-0588-0.

20. Consolvo, S.; McDonald, D.W.; Toscos, T.; Chen, M.Y.; Froehlich, J.; Harrison, B.L.; Klasnja, P.V.; LaMarca, A.; LeGrand, L.; Libby, R.; et al Activity Sensing in the Wild: A Field Trial of Ubifit Garden. In Proceedings of the 2008 CHI Conference on Human Factors in Computing Systems; Czerwinski, M., Lund, A.M., Tan, D.S., Eds.; ACM: New York, NY, USA, 2008; pp. 1797-1806. doi:10.1145/1357054.1357335.

21. WHO Fact Sheet: Tobacco. Available online: http://www.who.int/en/news-room/fact-sheets/detail/ tobacco (accessed on 8 May 2018).

22. Mollen, S.; Engelen, S.; Kessels, L.T.E.; van den Putte, B. Short and Sweet: The Persuasive Effects of Message Framing and Temporal Context in Antismoking Warning Labels. J. Health Commun. 2017, 22, $20-28$. doi:10.1080/10810730.2016.1247484.

23. Ghorai, K.; Akter, S.; Khatun, F.; Ray, P. mHealth for Smoking Cessation Programs: A Systematic Review. J. Personal. Med. 2014, 4, 412-423. doi:10.3390/jpm4030412.

24. Kocielnik, R.; Hsieh, G. Send Me a Different Message: Utilizing Cognitive Space to Create Engaging Message Triggers. In Proceedings of the 2017 ACM Conference on Computer Supported Cooperative Work and Social Computing (CSCW '17); ACM: New York, NY, USA, 2017; pp. 2193-2207. doi:10.1145/2998181.2998324.

25. Haskins, B.L.; Lesperance, D.; Gibbons, P.; Boudreaux, E.D. A systematic review of smartphone applications for smoking cessation. Transl. Behav. Med. 2017, 7, 292-299. doi:10.1007/s13142-017-0492-2.

26. Choi, J.; Noh, G.Y.; Park, D.J. Smoking Cessation Apps for Smartphones: Content Analysis with the Self-Determination Theory. J. Med. Internet Res. 2014, 16, e44. doi:10.2196/jmir.3061.

27. Karim, N.S.A.; AlHarbi, A.; AlKadhi, B.; AlOthaim, N. Mobile Application on Smoking Cessation Based on Persuasive Design Theory. Im Proceedings of the 21th Pacific Asia Conference on Information Systems (PACIS 2017), Langkawi, Malaysia, 16-20 July 2017.

28. Jamison, J.; Naughton, F.; Gilbert, H.; Sutton, S. Delivering Smoking Cessation Support by Mobile Phone Text Message: What Information do Smokers Want? A Focus Group Study. J. Appl. Biobehav. Res. 2013, 18, 1-23. doi:10.1111/jabr.12004.

29. Orji, R.; Moffatt, K. Persuasive technology for health and wellness: State-of-the-art and emerging trends. Health Inform. J. 2018, 24, 66-91.

30. Foster, D.; Linehan, C.; Kirman, B.; Lawson, S.W.; James, G. Motivating physical activity at work: Using persuasive social media for competitive step counting. In Proceedings of the 14th International Academic MindTrek Conference (MindTrek 2010); ACM: New York, NY, USA, 2010; pp. 111-116. doi:10.1145/1930488.1930510.

31. Khalil, A.; Abdallah, S. Harnessing social dynamics through persuasive technology to promote healthier lifestyle. Comput. Hum. Behav. 2013, 29, 2674-2681.

32. Young, M.M. Twitter Me: Using Micro-blogging to Motivate Teenagers to Exercise. In Proceedings of the International Conference on Design Science Research in Information Systems (DESRIST 2010); Springer-Verlag: Berlin/Heidelberg, Germany, 2010; Volume 6105, pp. 439-448. doi:10.1007/978-3-642-13335-0_30.

33. McClure, J.B.; Anderson, M.L.; Bradley, K.; An, L.C.; Catz, S.L. Evaluating an Adaptive and Interactive mHealth Smoking Cessation and Medication Adherence Program: A Randomized Pilot Feasibility Study. JMIR Mhealth Uhealth 2016, 4, e94. doi:10.2196/mhealth.6002. 
34. Pechmann, C.; Delucchi, K.; Lakon, C.M.; Prochaska, J.J. Randomised controlled trial evaluation of Tweet2Quit: A social network quit-smoking intervention. Tobacco Control 2017, 26, 188-194. doi:10.1136/tobaccocontrol-2015-052768.

35. Ubhi, H.K.; Michie, S.; Kotz, D.; Wong, W.C.; West, R. A Mobile App to Aid Smoking Cessation: Preliminary Evaluation of SmokeFree28. J. Med. Internet Res. 2015, 17, e17. doi:10.2196/jmir.3479.

36. Google Play. QuitNow! Dejar de Fumar. Available online: https://play.google.com/store/apps/details? $\mathrm{id}=$ com.EAGINsoftware.dejaloYa (accessed on 2 May 2017)

37. Google Play. 7 Weeks-Habit \& Goal Tracker. Available online: https://play.google.com/store/apps/ details?id=com.ryan.brooks.sevenweeks.app (accessed on 2 May 2017)

38. Google. Google APIs for Android: DetectedActivity. Available online: https://developers.google.com/ android/reference/com/google/android/gms/location/DetectedActivity (accessed on 5 September 2018)

39. Fagerström, K.O. Measuring degree of physical dependence to tobacco smoking with reference to individualization of treatment. Addict. Behav. 1978, 3, 235-241.

40. Ferrari, A. Digital Competence in Practice: An Analysis of Frameworks; Technical Report EUR 25351 EN; Research Centre of the European Commission: Brussels, Belgium, 2012.

41. Tullis, T.; Albert, W. Measuring the User Experience: Collecting, Analyzing, and Presenting Usability Metrics; Morgan Kaufmann Publishers Inc.: Burlington, MA, USA, 2008.

42. Cronbach, L.J. Coefficient alpha and the internal structure of tests. Psychometrika 1951, 16, $297-334$. doi:10.1007/BF02310555.

43. DeVon, H.A.; Block, M.E.; Moyle-Wright, P.; Ernst, D.M.; Hayden, S.J.; Lazzara, D.J.; Savoy, S.M.; Kostas-Polston, E. A Psychometric Toolbox for Testing Validity and Reliability. J. Nurs. Scholarsh. 2007, 39, 155-164. doi:10.1111/j.1547-5069.2007.00161.x.

(C) 2018 by the authors. Licensee MDPI, Basel, Switzerland. This article is an open access article distributed under the terms and conditions of the Creative Commons Attribution (CC BY) license (http://creativecommons.org/licenses/by/4.0/). 